\title{
Analytical Calculation of the Impedance of Lossy Power/Ground Planes
}

\author{
Bertalan Eged and László Balogh
}

\begin{abstract}
Power and ground planes are required to have low impedance over a wide range of frequencies. Parallel ground and power planes in multilayer printed-circuit boards exhibit multiple resonances, which increase the impedance. Dissipative loading of the radial transmission line structure of the planes reduces the resonance peaks. The dissipative loads can be realized by resistors distributed on the surface or the edges of plain pairs and lossy dielectric material can be applied for distributed loading, and the characterization of the impedance by simulation is very important during the design phase. Measurement results are compared with analytical solutions and simulation results. Furthermore, in this paper, a new algorithm is given for the more efficient calculation of the impedance of power distribution structures.
\end{abstract}

Index Terms-Impedance calculation, lossy power/ground planes, resonance effect.

\section{INTRODUCTION}

$\mathbf{T}$ HE faster the bus signaling, the faster are slopes and the transients in a power-distribution network. Therefore, a proportionally wider bandwidth is required. A high-end system today with single-ended signaling may have 10-A total transient current in the signal-return path of a bus, and may require $50-\mathrm{mV}$ maximum ripple on the power-distribution network. This converts into $5 \mathrm{~m} \Omega$ of required power-distribution impedance. With a $0.3 \ldots 0.6 \mathrm{~ns}$ signal transition time, the necessary bandwidth for the power-distribution impedance amounts to $0.5-1 \mathrm{GHz}$. To avoid noise, the power-distribution network (i.e., the power-distribution planes) must exhibit low enough impedance over the full bandwidth of signals. Therefore, during the design phase a fast and accurate characterization of impedance should be done.

This paper reviews the known methods, and proposes a new and more effective one. Finally, simulation and measurement results are shown for validation.

\section{Characterization OF POWER-GROUND DISTRIBUTION SYSTEM}

For digital electronics below the megahertz clock-frequency range, individual traces or metal bus bars were sufficient to distribute ground and power.

Manuscript received June 15, 2001; revised August 15, 2003.

The authors are with the Department of Microwave Telecommunications and the Department of Measurement and Information Systems, Budapest University of Technology and Economics, Budapest, Hungary (e-mail: beged@mht.bme.hu; blgh@mht.bme.hu).

Digital Object Identifier 10.1109/TIM.2003.820471

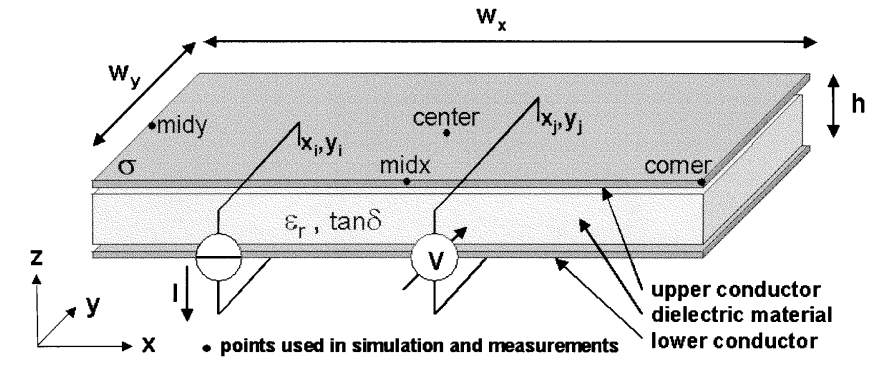

Fig. 1. Pair of parallel planes in the power distribution system (PDS).

Power and ground planes in a multilayer printed circuit board (PCB) of the high-speed application may be considered as twodimensional transmission lines, where both the $x$ and $y$ dimensions are longer than one tenth of the shortest wavelength of interest. Throughout this paper, we assume that the separation $h$ of the planes along the $z$ axis is still negligible compared to the shortest wavelength.

In this structure, the different impedances are defined in the following way (see Fig. 1):

- the impedance of the power distribution system is defined by $Z_{i, j}=\left(V_{x_{j}, y_{j}}\right) /\left(I_{x_{i}, y_{i}}\right)$;

- this is called self impedance if $i=j$, and transfer impedance otherwise.

\section{A. Analytical Expression of Lossless Power-Ground Plane Impedances}

In contrast to signal traces where the signal travels along the axis of the signal conductor, the wave generated by an injected signal between the planes launches a radially expanding wave. Two-dimensional (2-D) transmission lines are therefore also referred to as radial transmission lines. The self and transfer impedances of radial transmission lines with rectangular or circular shapes can be analytically calculated. Impedances of square-shaped parallel planes are widely analyzed in the literature for planar microwave circuits and printed antennas. Analytical formulation is given, e.g., in [1], [2]. Assuming infinitesimally small port sizes, open boundaries at the edges, and a pair of parallel, rectangular planes with side dimensions $w_{x}$ and $w_{y}$ along the $x$ and $y$ axes, with plane separation (dielectric height) of $h$ along the $z$ axis, the generalized transfer 
impedance between ports $i$ and $j$ (at coordinates $x_{i}, y_{i}$, and $x_{j}, y_{j}$ respectively) can be written as [3]

$$
\begin{aligned}
Z_{i, j} & =\frac{\mu h}{w_{x} w_{y}} \sum_{m=0}^{\infty} \sum_{n=0}^{\infty} \frac{\chi_{m n}^{2} j w}{k_{m}^{2}+k_{n}^{2}-k^{2}} C, \\
C & =\cos k_{m} x_{i} \cos k_{n} y_{i} \cos k_{m} x_{j} \cos k_{n} y_{j},
\end{aligned}
$$

where $m$ represents the $m$ th mode associated with the $x$ dimensions, $n$ represents the $n$th mode associated with the $y$ dimensions, $k$ represents the real wavenumber for lossless case, $k=\omega \sqrt{\mu \epsilon}, k_{m}=m \pi w_{x}, k_{n}=n \pi w_{y}$. The constant $\chi_{m n}=1$ for $m=0$ and $n=0, \sqrt{2}$ for $m=0$ or $n=0,2$ for $m \neq 0$ and $n \neq 0$. In a low-loss case, $k$ is complex: $k=k_{r}-j k_{i}$, where $k_{r}=k$ above and $k_{i}=(k r / 2)(\tan (\delta)+(r / h))$, where $\tan (\delta)$ is the loss tangent of the dielectric, and $r$ is the skin depth in the metal plane.

The analytical expression is not limited by finite spatial granularity like the transmission line grid model, but it has a double infinite series, which for practical calculations must be truncated, leading to an error in calculation [4]. The above $Z(j w)$ expression is well for numerical calculation, but is not adequate for circuit simulation where the planes are simulated with the whole part of electronic devices. For circuit simulations, either a macro model can be generated [5], or an electrical equivalent circuit of the pair of planes can be formed.

\section{B. Transmission Line Grid Equivalent Circuit Model of the Ideal Power-Ground Plane}

A pair of parallel planes can be simulated by an equivalent circuit of a grid of transmission lines, as described in e.g., [6], [7]. The low-frequency equivalent components of the planes can be derived from a quasistatic model. We assume a pair of rectangular planes with dimensions of $w_{x}$ and $w_{y}$. First, we define the $u$ size of the square unit grid cell, which should be equal to or less than $10 \%$ of the shortest wavelength of interest. The $u$ cell size is selected such that we have an integer $N_{x}$ and $N_{y}$ number of cells along the $x$ and $y$ axes, respectively. For every unit square of the planes with side dimensions of $u$, plane separation (dielectric height) of $h$, the propagation delay $t_{\mathrm{pd}}$ along the edge of the unit cell and the static plane capacitance $C$ can be calculated as

$$
\begin{aligned}
t_{\mathrm{pd}} & =u \frac{1}{c} \sqrt{\epsilon_{r}}=u \sqrt{\epsilon_{r}} \sqrt{\epsilon_{0} \mu_{0}} \\
C & =\frac{u^{2}}{h} \epsilon_{r} \epsilon_{0} .
\end{aligned}
$$

From the capacitance and delay, an equivalent $L$ inductance and $Z_{0}$ characteristic impedance of the unit cell can be calculated

$$
\begin{aligned}
L & =\frac{t_{\mathrm{pd}}}{C}=h \mu_{0} \\
Z_{0} & =\sqrt{\frac{L}{C}}=\frac{h}{u} \frac{1}{\sqrt{\epsilon_{r}}} \sqrt{\frac{\mu_{0}}{\epsilon_{0}}} .
\end{aligned}
$$

In the above expressions, all input and output parameters are in SI units, $\epsilon_{0}=8.86 \cdot 10^{-12}[(\mathrm{As} / \mathrm{Vm})]$ is the dielectric constant in vacuum, $\mu_{0}=4 \cdot 10^{-7}[(\mathrm{H} / \mathrm{m})]$ is the permeability of vacuum.

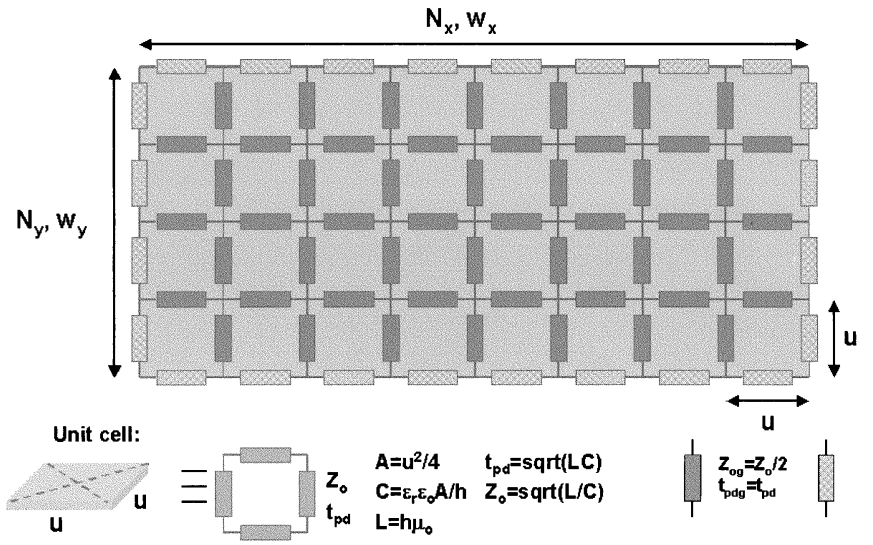

Fig. 2. Equivalent circuit representation of parallel conductive planes with a rectangular grid of transmission lines.
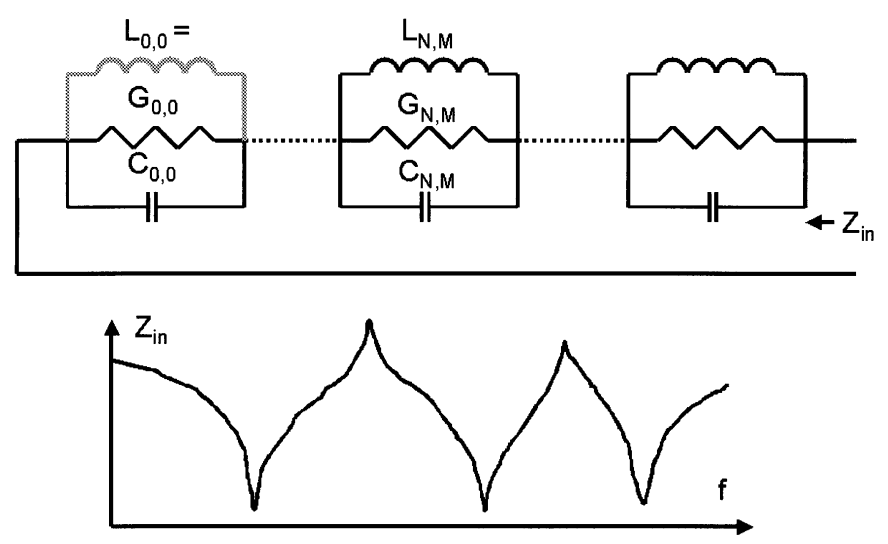

Fig. 3. Equivalent circuit describing the impedance of a given point of the plain.

The unit cells are replaced by four transmission lines along the edges of the unit cells, (Fig. 2), each transmission line representing the same delay and only one quarter of the area, thus having an impedance of $2 Z_{0}$. Inside the equivalent grid, where the sides of unit cells meet, the capacitances of transmission lines are doubled, reducing the characteristic impedance to $\left(2 Z_{0} / \sqrt{2}\right)$. Whereas along the outer edges, the unit-cell transmission lines are standing alone. The parameters for the edge $\left(Z_{0 e}, t_{\text {pde }}\right)$ and grid $\left(Z_{0 g}, t_{\text {pdg }}\right)$ transmission lines are [8]

$$
\begin{array}{ll}
Z_{0 g}=\sqrt{2} Z_{0} & t_{\mathrm{pdg}}=\frac{t_{\mathrm{pd}}}{\sqrt{2}} \\
Z_{0 g}=2 Z_{0} & t_{\mathrm{pde}}=\frac{t_{\mathrm{pd}}}{\sqrt{2}} .
\end{array}
$$

The correction factors $\sqrt{2}$ in the delays are used to match the equivalent circuit's delay along the $x$ and $y$ axes [9]. Alternative equivalent circuits may use lossless LC ladder [10] or lossy transmission lines [9], [11] to represent of each transmission-line segment. For all simulations presented in this paper, lossless transmission line grids were used.

Note that the grid takes account of some extent, the effect of the edge discontinuity by using twice the characteristic impedances of transmission lines along the edges. The transmission line model can be used easily for simulating the power planes and the other components of the circuit including the 


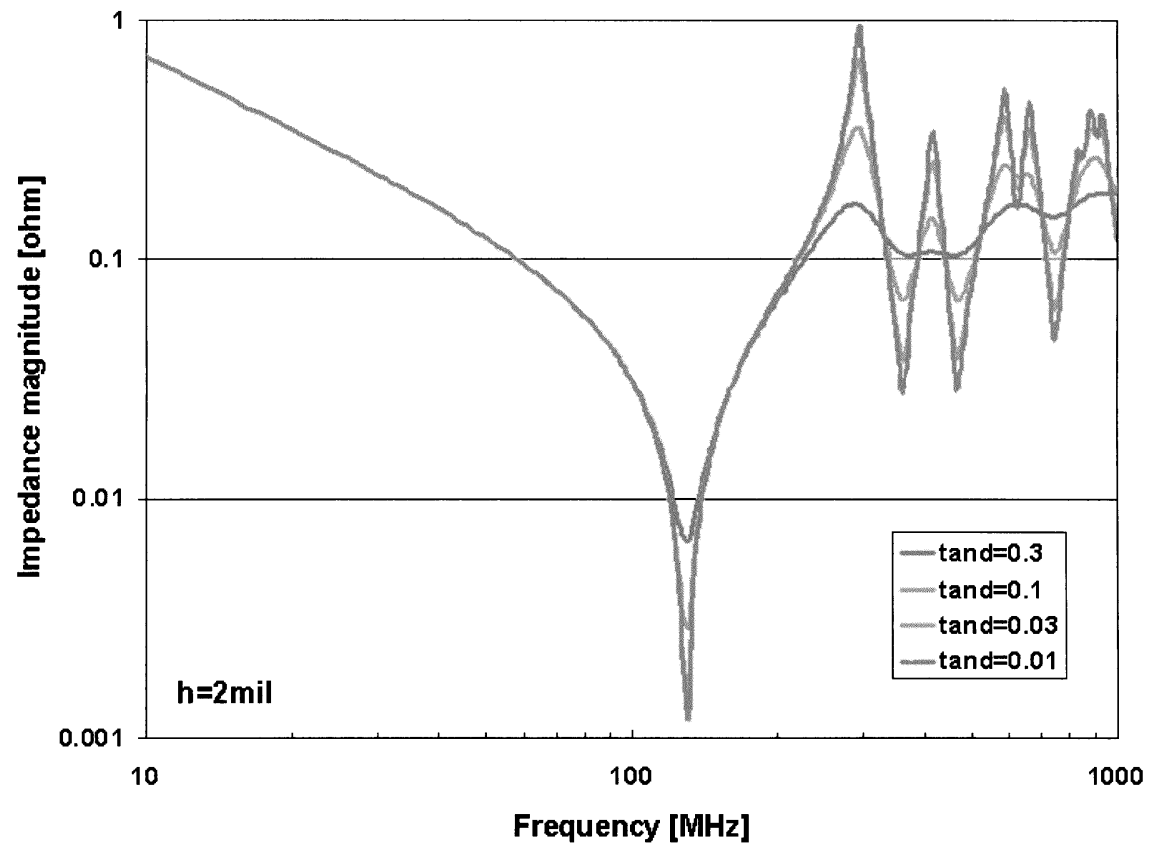

Fig. 4. Calculated impedance profile of a $10^{\prime \prime} \times 10^{\prime \prime}$ plan pairs with different dielectric loss.

dissipative edge termination [11], which is used for reducing the effect of the resonance behavior. The price to be paid for this feature of the model is the spatial granularity of the transmission line grid.

\section{Analytical Calculations of Lossy Plain Pairs}

The calculation of the impedance profile in the case of lossy dielectric material can be based on the equivalent circuit of the impedance (Fig. 3) at a given point [1]. The equivalent circuit consists of an infinite number of serially connected resonators. The loss of the dielectric material is represented by the $G_{N, M}$ admittance in the circuit. The impedance elements of the equivalent circuit (rectangular plane with dimensions $w_{x}, w_{y}$ and point of measurement $x_{i}, y_{i}$ ) are the following:

$$
\begin{aligned}
Z_{\text {in }}(f) & =\sum_{n=0}^{N} \sum_{m=0}^{M} \frac{1}{j 2 \pi f C_{m, n}-j \frac{1}{2 \pi f L_{m, n}}+G_{m, n}}, \\
C_{m, n} & =\frac{\epsilon_{r} \in w_{x} w_{y}}{2 h} \frac{1}{F_{m, n}}, \\
L_{m, n} & =\frac{2 \mu h F_{m, n}}{w_{x} w_{y}\left(k_{m}^{2}+k_{n}^{2}\right)}, \\
G_{m, n} & =\frac{2 \pi f_{0 m, n} C_{m, n}}{Q_{0}(f)}, \\
F_{m, n} & =\cos \left(k_{m} x_{i}\right) \cos \left(k_{n} y_{i}\right) \\
f_{0 m, n} & =\frac{\sqrt{\left(\frac{m}{w_{x}}\right)^{2}+\left(\frac{n}{w_{y}}\right)^{2}}}{2 \sqrt{\epsilon \mu} \sqrt{\epsilon_{r}}} \\
Q_{0}(f) & =\frac{1}{Q_{d}(f)^{-1}+Q_{c}(f)^{-1}}, \\
Q_{d}(f) & =\frac{1}{\tan \delta} Q_{c}(f)=\frac{h}{r(f)} \\
r(f) & =\sqrt{\frac{2}{2 \pi f 10^{6} \mu \sigma}} .
\end{aligned}
$$

The known impedance profile and its different characteristics have also been introduced, and [4] describes this type of equivalent circuit in detail. Calculation with the above described method was done for a $10^{\prime \prime}$ by $10^{\prime \prime}$ power/ground plane pair. The impedance profile at the corner of the plane was calculated with different dielectric losses. The results can be seen in Fig. 4. As it was pointed out in [12], the thickness of the dielectric material is important parameter to introduce a wide-band low impedance power distribution system. The impedance was calculated for different dielectric thickness, too. The results are in Fig. 5. and they are very similar to the simulated ones in [12].

\section{EFFECT OF ThE Mode Number Truncation}

In the practice for speeding up the calculations, the mode numbers are truncated. The effect of this truncation is reported and evaluated in [4]. The plane-impedance expression contains a double series of second-order terms. These terms accurately describe the poles (peaks) in the impedance profile, and the frequencies of the peaks do not change as we add or remove terms. The minimum of the impedance profile, however, does change as more terms are added to the series. More importantly, beyond the frequency of the last pole, the truncated series yields an impedance of capacitive downslope, as opposed to the inductive upslope of the plane impedance at high frequencies.

The truncation of the two dimensional modal space can be done in different ways. Some practical ways are illustrated in Fig. 6.

The published calculation methods use the rectangular truncation, but in [13] the elliptical truncation is used and the convergence of this method is reported. The main advantage of 


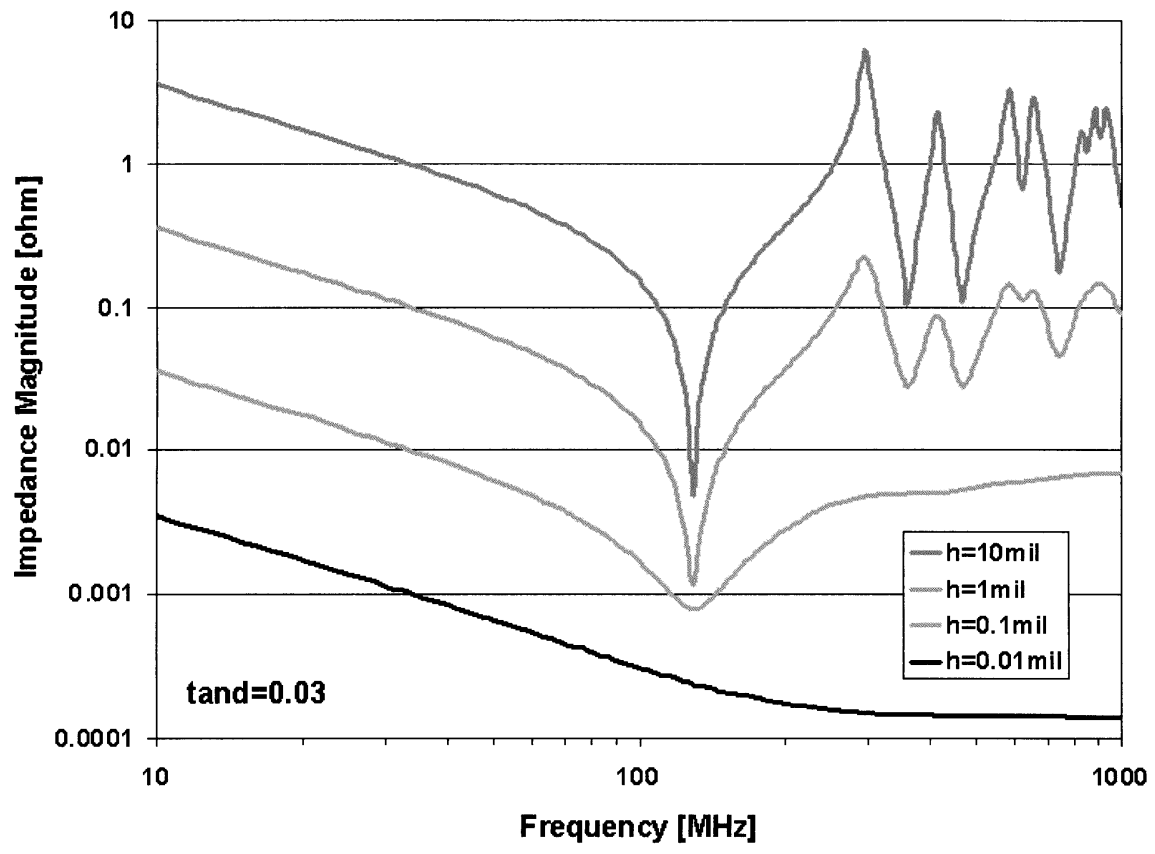

Fig. 5. Calculated impedance profile of a $10^{\prime \prime} \times 10^{\prime \prime}$ plan pairs with different dielectric thickness.

the calculation compared to transmission line grid simulation is that the truncation of the double infinite series can be used. With a different truncation method, the speed of calculation can be increased proportionately to the area of the truncated modal space. However, using truncation, a trade-off has to be made between calculation time and accuracy.

The calculation program is based on the formulas of concentrated elements, lossy equivalent circuit. Rectangular, elliptic, linear, and inverse elliptic mode truncations were implemented, and the calculations of first resonant frequencies were evaluated regarding the speed and accuracy.

As it can be seen in Figs. 7 and 8, the differences of the frequencies of first resonants comparing to the rectangular mode truncation are less than $0.75 \%, 2.5 \%, 5.9 \%$ for elliptic, linear, and inverse elliptic truncations, respectively.

\section{Measurement Results}

To correlate calculated and measured impedances, a $10 \times 10$-in square pair of planes was selected with 31 mil FR4 dielectric material.

Self impedances were measured with HP8752A vector-network analyzer in the $1 \mathrm{MHz}-1 \mathrm{GHz}$ range at the center, corner and edge of the test board (Fig. 9). The probe connections, calibrations, and conversions from $\mathrm{S}$ parameters to impedances were according to [14].

To improve the measurement accuracy at low impedance readings, two-port $\mathrm{S} 21$-based self-impedance measurement was used. The S21 parameter readings were converted to self and

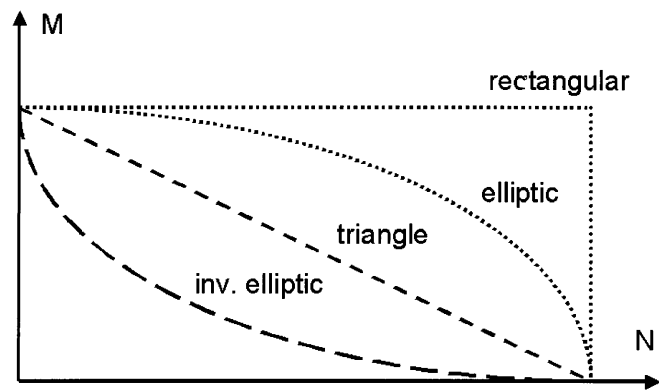

Fig. 6. Possible ways for truncating the mode numbers.

transfer impedance values by the $Z=25 S_{21}$ approximative formula [14].

\section{NOVELTIES}

This paper introduces a new procedure for calculating the impedance in the case of lossy power/ground planes based on discrete component equivalent circuit. This method can be used for the calculations of distributed loss applied in the dielectric material and metallization, too.

The method can be used for speeding up the calculation by allowing different types of mode truncation. The speed and the error of the calculation of the resonance frequencies were evaluated and presented for rectangular and elliptic truncation of mode numbers. Simulation based on the discrete component equivalent circuit should be used to allow the usage of the mode truncation feature. 


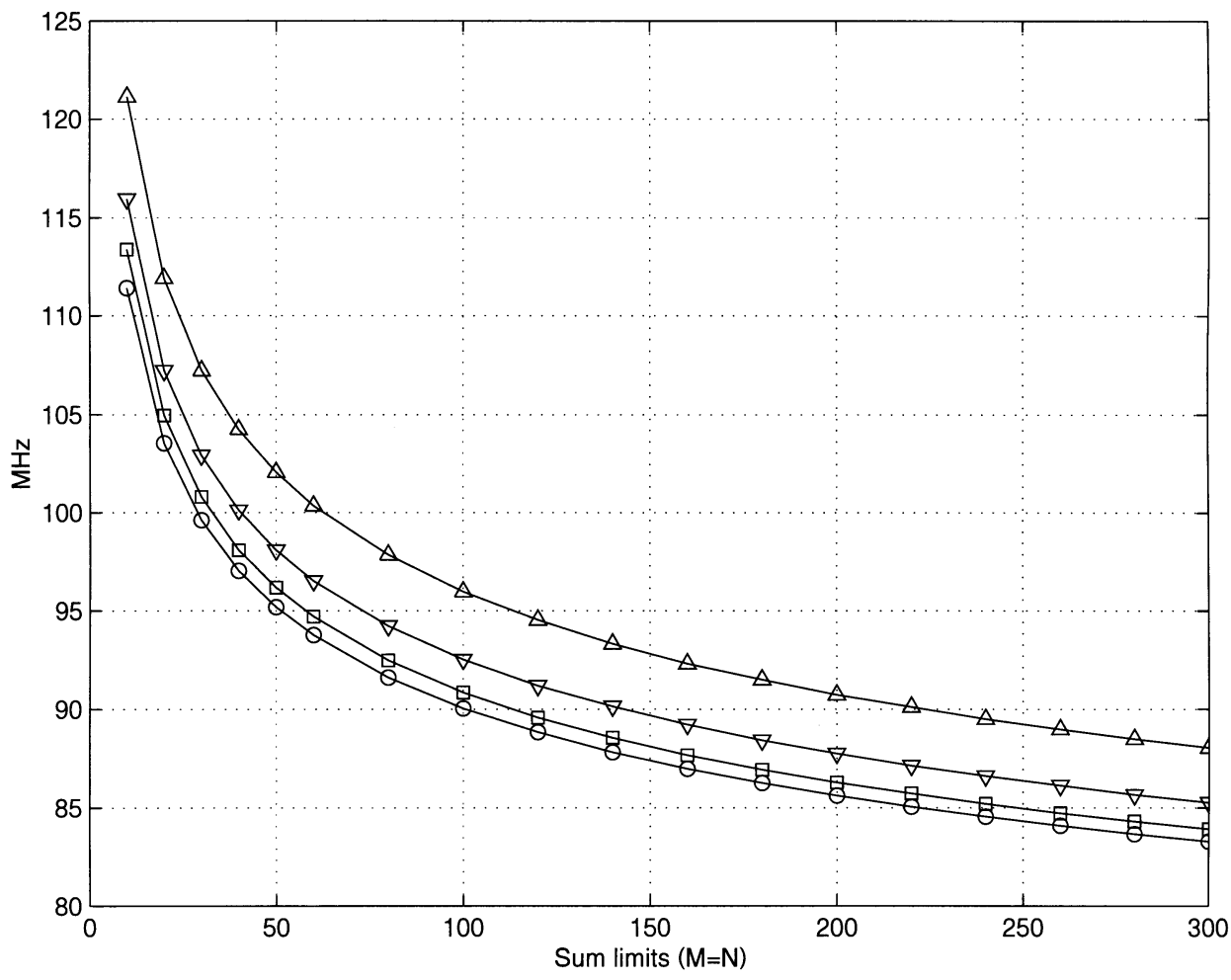

Fig. 7. Calculated first resonant frequency using different truncation modes (circle: rectangular, square: elliptic, triangle down: linear, triangle up: inverse elliptic).

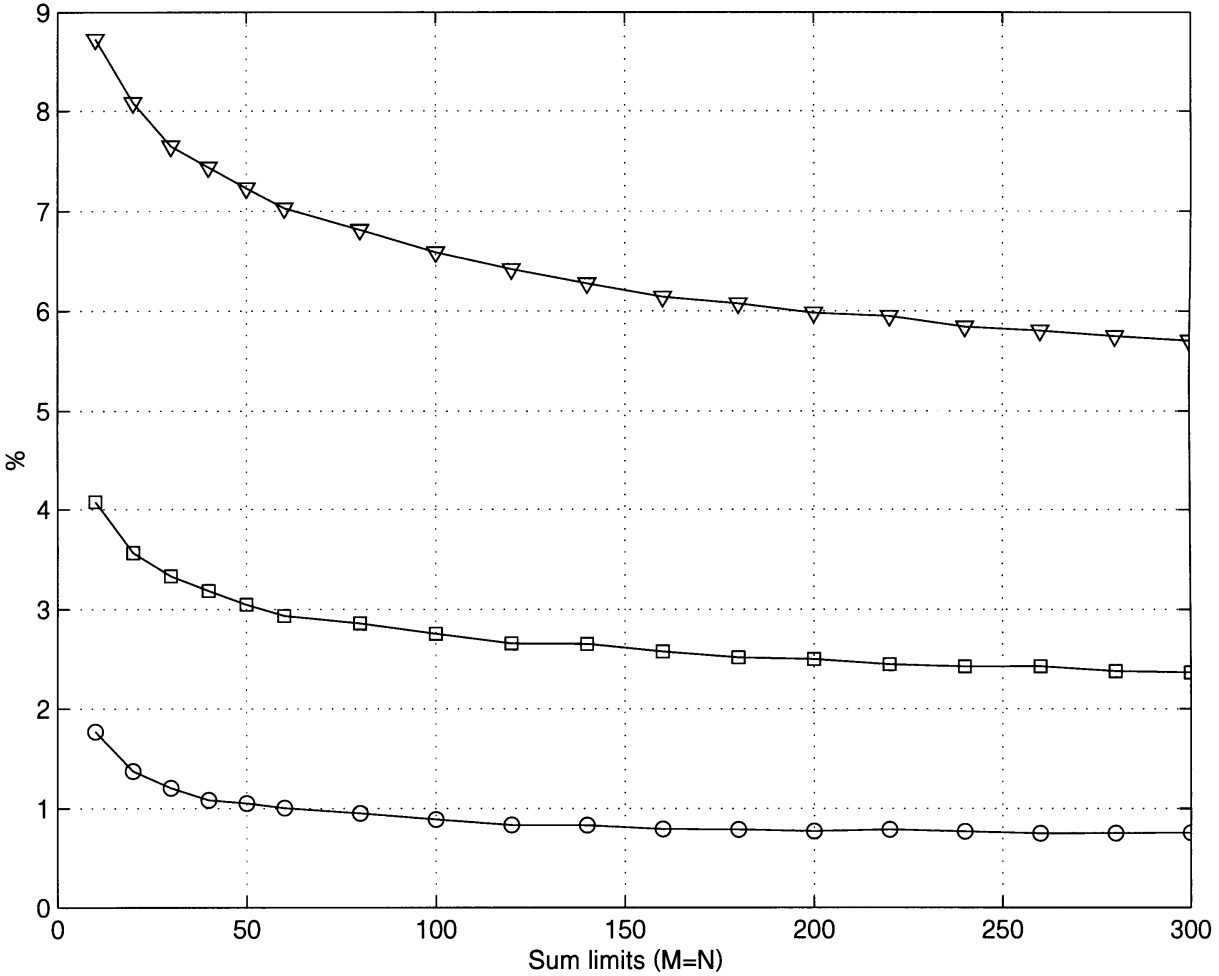

Fig. 8. Relative difference between resonant frequencies calculated with different truncations (circle: elliptic, square: linear, triangle down: inverse elliptic). 


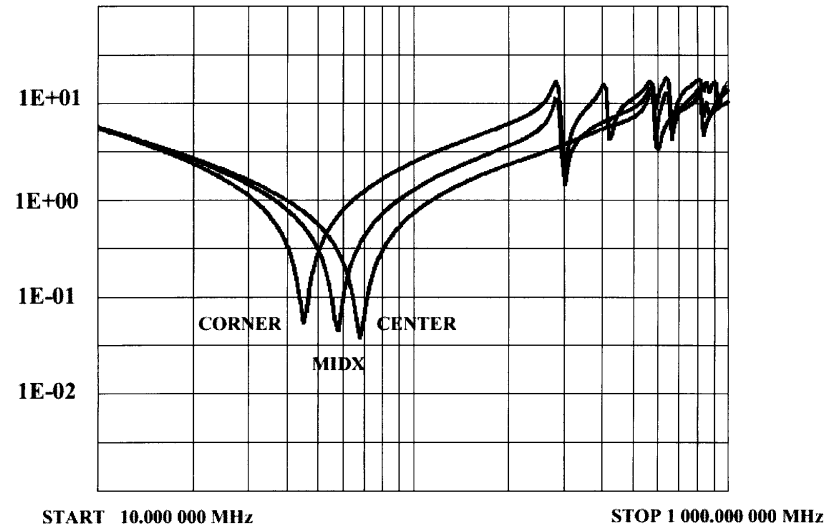

Fig. 9. Measure self impedances of the power plane.

\section{REFERENCES}

[1] T. Miyoshi and T. Okoshi, "The planar circuit-An approach to microwave integrated circuit," IEEE Trans. Microwave Theory Tech., vol. 20, pp. 245-252, Apr. 1972.

[2] J. W. Mink and K. R. Carver, "Microstrip antenna technology," IEEE Trans. Antennas Propagat., pp. 2-24, Jan. 1981.

[3] T. Itoh, Numerical Techniques for Microwave and Millimeter-Wave Passive Structures. New York: Wiley, 1989.

[4] I. Novák, "Accuracy considerations of power-ground plane models," in Proc. 8th Topical Meeting on Electrical Performance of Electronic Packaging, San Diego, CA, Oct. 1999, pp. 153-156.

[5] N. Na, "Madhavan swaminathan, "modeling and simulation of planes in electronic packages for ghz systems"," in Proc. Int. Conf. Computer Design, Oct. 1999, pp. 149-152.

[6] P. Bajor and E. Leroux, "Modeling of power planes for electrical simulations," in Proc. Wroclaw EMC Symp., pp. 664-668.

[7] A. Barber and K. Lee, "Modeling and analysis of multichip module power supply planes," IEEE Trans. Compon., Packag., Manufact. Technol. B, vol. 18, pp. 628-639, Nov. 1995

[8] L. Smith, R. Anderson, and T. Roy, "Power plane spice models and simulated performance for materials and geometries," IEEE Trans. Adv Packag., 2001.
[9] K. Lee et al., "Accurate power supply and ground plane pair models," in Proc. 7th Topical Meeting on Electrical Performance of Electronic Packaging, Oct., pp. 163-166.

[10] D. W. Forehand, C. B. O'Sullivan, and L. D. Smith, "Developing a decoupling methodology with spice for multilayer printed circuit boards," in Proc. Int. Symp. Electromagnetic Compatibility, Denver, CO, Aug. 1998, pp. 562-565.

[11] I. Novák, "Reducing simultaneous switching noise and emi on ground/power planes by dissipative edge termination," IEEE Trans. CPMT, vol. 22, pp. 274-283, Aug. 1999.

[12] — "Design considerations and modeling accuracy of lossy power ditribution networks," IEEE Trans. CPMT, vol. 23, Aug. 2000.

[13] B. K. Gilbert, G.-T. Lei, and R. W. Techentin, "High-frequency characterization of power/ground-plane structures," IEEE Trans. Microwave Theory Tech., vol. 47, pp. 562-569, May 1999.

[14] I. Novák, "Probes and setup for measuring power-plane impedances with vector network analyzer," in Proc. High-Performance System Design Conference, Santa Clara, CA, 1999.

Bertalan Eged was born in Eger, Hungary, in 1966. He received the M.S. degree in electrical engineering from the Technical University of Budapest, Budapest, Hungary, in 1990.

He is a Senior Lecturer with the Department of Microwave Telecommunication, Budapest University of Technology and Economics. From 1997 to 1999, he was the Leader of the Hardware Design Group of ALICE DDL project in CEREN, Geneva, Switzerland. He is interested in signal integrity problems of high-speed digital circuits, hardware implementation, and protocol layers of software defined radio solutions.

László Balogh was born in Békéscsaba, Hungary, in 1977. He received the M.Sc. degree in electrical engineering from the Budapest University of Technology and Economics, Budapest, Hungary, in 2000. He is currently pursuing the Ph.D. degree.

His research interests include system identification, signal processing, high frequency ground planes, and related applications. 